\section{Diet and gender influence survival of transgenic Berkley sickle cell mice}

The prevalence of sickle cell disease (SCD) is highest in regions in which there is global malnutrition with high infant and early childhood mortality. ${ }^{1,2}$ In sub-Saharan Africa, 5-year olds are at the highest risk of death. ${ }^{1}$ In tribal communities of India, $20 \%$ of children with SCD die by the age of 2 years and $30 \%$ die before reaching adulthood. ${ }^{2}$ Additionally, individuals with SCD may suffer from disease-related malnutrition as a result of increased demands for absorbed nutrients, caused by multiple pathological factors including higher resting basal metabolic rate. ${ }^{3,4}$ Deficiencies of many nutrients, including vitamins, zinc, magnesium, and fatty acids are well-documented in SCD. ${ }^{3}$ Nutritional deficiencies may begin during gestation because of poor parental diet, which adds to the existing challenge of examining the effect of diet on survival of individuals with SCD. Transgenic mouse models of SCD including homozygous Berkley (HbSS-BERK) expressing exclusively human sickle hemoglobin replicate the pathophysiology of human SCD and provide an opportunity to examine the effect of dietary interventions across generations. ${ }^{5}$ We examined whether a diet enriched in protein, amino acids, fatty acids, and specific critical micronutrients (Table 1) fed to parents and/or offspring would improve the survival of offsprings with SCD.

We used protocols approved by the Institutional Animal Care and Use Committee of the University of Minnesota. HbSS-BERK mice offspring (henceforth called pups) expressing $>99 \%$ human sickle hemoglobin were used. ${ }^{5}$ Because homozygous BERK female mice often do not breed/survive pregnancy, ${ }^{6}$ we used heterozygous BERK female (HbAS-BERK) and male HbSS-BERK as breeding pairs (Figure 1). Breeders and pups were randomly assigned to either a sickle diet (SD) or regular diet (RD) (Table 1) to differentiate groups by pup diet, parental diet, and gender (Figure 1). After phenotyping, only the homozygous pups were included in the study. Breeder pairs were divided into two dietary groups, SD and $\mathrm{RD}$, as shown in Figure 1. Compared to the RD, the $\mathrm{SD}$ has a higher content of protein and fat calories and contains higher amounts of specific minerals, vitamins, amino acids, and omega-3-fatty acids (Table 1). Pups were weaned 3 weeks after birth and fed the SD for 10 days after weaning and then randomly assigned to either the SD or RD until 150 days of age. A multivariate analysis was performed on the relationship between rates of survival and the following factors of interest: pup gender, pup diet, and parental diet. The outcome variable of interest, survival in days, was examined using KaplanMeier plots of estimated survival and Cox regression to obtain hazard ratios (HR) with $95 \%$ confidence intervals (95\% CI). For Cox regression, the significance of individual factors in multivariate analysis and the significance of overall survival differences were based on Wald and logrank tests, respectively. Data were analyzed using the 'survival' package in 'R 3.4.0' (R Foundation for Statistical Computing; Vienna, Austria). $P$ values $<0.05$ were considered statistically significant.

We first studied the effect of diet on survival of HbSSBERK pups. Of 601 pups, 482 were given the SD and 119 were given the RD. At 150 days, $77 \%$ of pups on the SD and $51 \%$ of pups on the $\mathrm{RD}$ were alive (Figure 2A). According to the multivariate analysis, pups fed the SD had significantly prolonged survival compared to pups on the RD (HR: 0.3988; 95\% CI: $0.2860-0.5561$;

\section{$\left.P=6.03 \times 10^{-8}\right)$.}

Dietary supplementation with individual nutrients has been shown to attenuate pathogenic mechanisms and reduce morbidity associated with SCD. A high protein diet attenuated ischemic end-organ damage, increased muscle mass, bone mineral density, and grip strength, and decreased vascular leakage in HbSS-BERK or Townes sickle mice., ${ }^{7,8}$ Omega-3-fatty acid supplementation decreased NF- $\kappa \mathrm{B}$ activation, adhesion molecule expression, and inflammatory markers and reduced the frequency of vaso-occlusive crises and transfusion requirements in patients with SCD. ${ }^{9,10}$ Zinc supplementation improved growth and decreased the incidence of infection. ${ }^{11}$ Folic acid is critical for DNA synthesis and does not have body stores. A compensatory increase in erythropoiesis in SCD results in folate deficiency due to over-utilization. ${ }^{12}$ In HbSS-BERK mice, arginine supplementation markedly enhanced nitric oxide bioavailability, and decreased both hemolysis and oxidative stress. ${ }^{13}$ Arginine supplementation alleviated pain induced by vaso-occlusive crises and decreased narcotic use by $>50 \%$ in a randomized placebo-controlled clinical trial. ${ }^{14}$ In a comparison with placebo administration, L-glutamine supplementation $(0.6 \mathrm{~g} / \mathrm{Kg} /$ day $)$ reduced the average cumulative recurrence of vaso-occlusive crises but significantly increased musculoskeletal, abdominal and chest pain in patients with SCD in a phase III clinical trial. ${ }^{15}$ The SD includes these nutrients but in significantly smaller amounts, which may synergize to meet the nutritional requirements in SCD without causing off-target effects. Therefore, supplementation of diet with a combination of multiple nutrients may be more beneficial than the use of individual nutrients at high doses.

We next studied the effects of gender on survival of HbSS-BERK pups. We compared the survival of 338 female pups and 263 male pups independent of their

Table 1. Composition of the sickle diet and the regular diet.

\begin{tabular}{lcc}
\hline Contents & Sickle Diet & Regular Diet \\
Protein (\% weight) & 26.4 & 18.6 \\
Fat (\% weight) & 11.1 & 6.2 \\
\hline Carbohydrates (\% weight) & 62.5 & 44.2 \\
Protein (\% calories) & 27.5 & 24 \\
\hline Fat (\% calories) & 26 & 18 \\
Carbohydrates (\% calories) & 46.5 & 58 \\
\hline Arginine (\%) & 2.52 & 1 \\
Aspartic acid (\%) & 2.53 & 1.4 \\
\hline Glutamic acid (\%) & 5.16 & 3.4 \\
Magnesium (\%) & 0.48 & 0.2 \\
\hline Sulfur (\%) & 0.29 & 0 \\
Zinc (mg/kg) & 225 & 70 \\
Vitamin A (IU/g) & 33 & 15 \\
Vitamin D3 (IU/g) & 3.3 & 1.5 \\
\hline Vitamin E (IU/kg) & 200 & 110 \\
Folic acid (mg/kg) & 8 & 4 \\
\hline Vitamin B12 $(\mu \mathrm{g} / \mathrm{kg})$ & 60 & 80 \\
Choline chloride $(\mathrm{mg} / \mathrm{kg})$ & 2200 & 1200 \\
\hline
\end{tabular}

The major components of the individual diets are listed above.The sickle diet was the Sickle Cell Mouse Diet (59M3) (TestDiet, St. Louis, MO, USA). A complete list of its components can be found at: www.testdiet.com. The reegular diet was the Teklad Global 18\% Protein Rodent Diet (Harlan Laboratories; Madison,WI, USA) A complete list of its components can be found at:www.envigo.com/resources/datasheets/2018-datasheet-0915.pdf. 


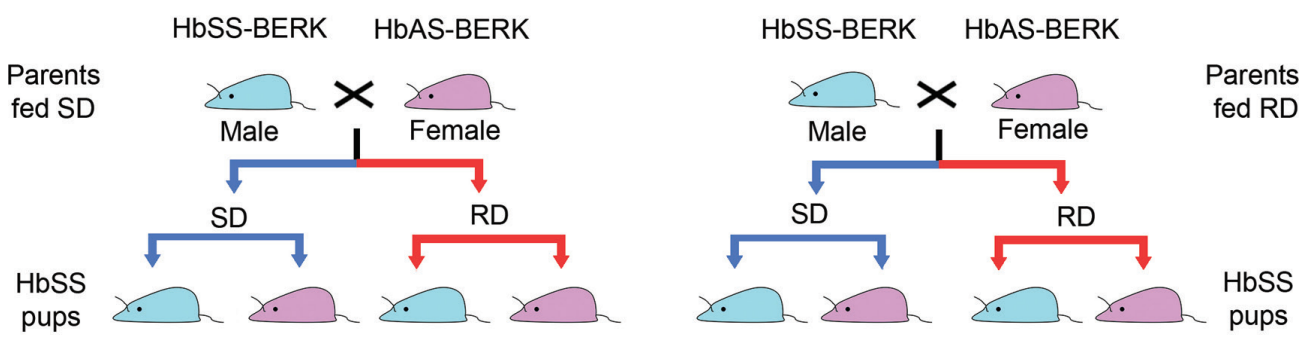

Figure 1. Breeding strategy. Parent pairs comprised male homozygous (HbSS-BERK) and female hemizygous (HbAS-BERK) sickle mice. After phenotyping and genotyping, only homozygous HbSS pups were included in the study. Breeder pairs were divided into two groups according to the diet they were fed: the sickle diet (SD) or the regular diet (RD). Contents of the SD and RD are specified in Table 1. Pups were weaned 3 weeks after birth, fed the SD for 10 days after weaning and then randomly assigned to either the SD or RD until 150 days of age.
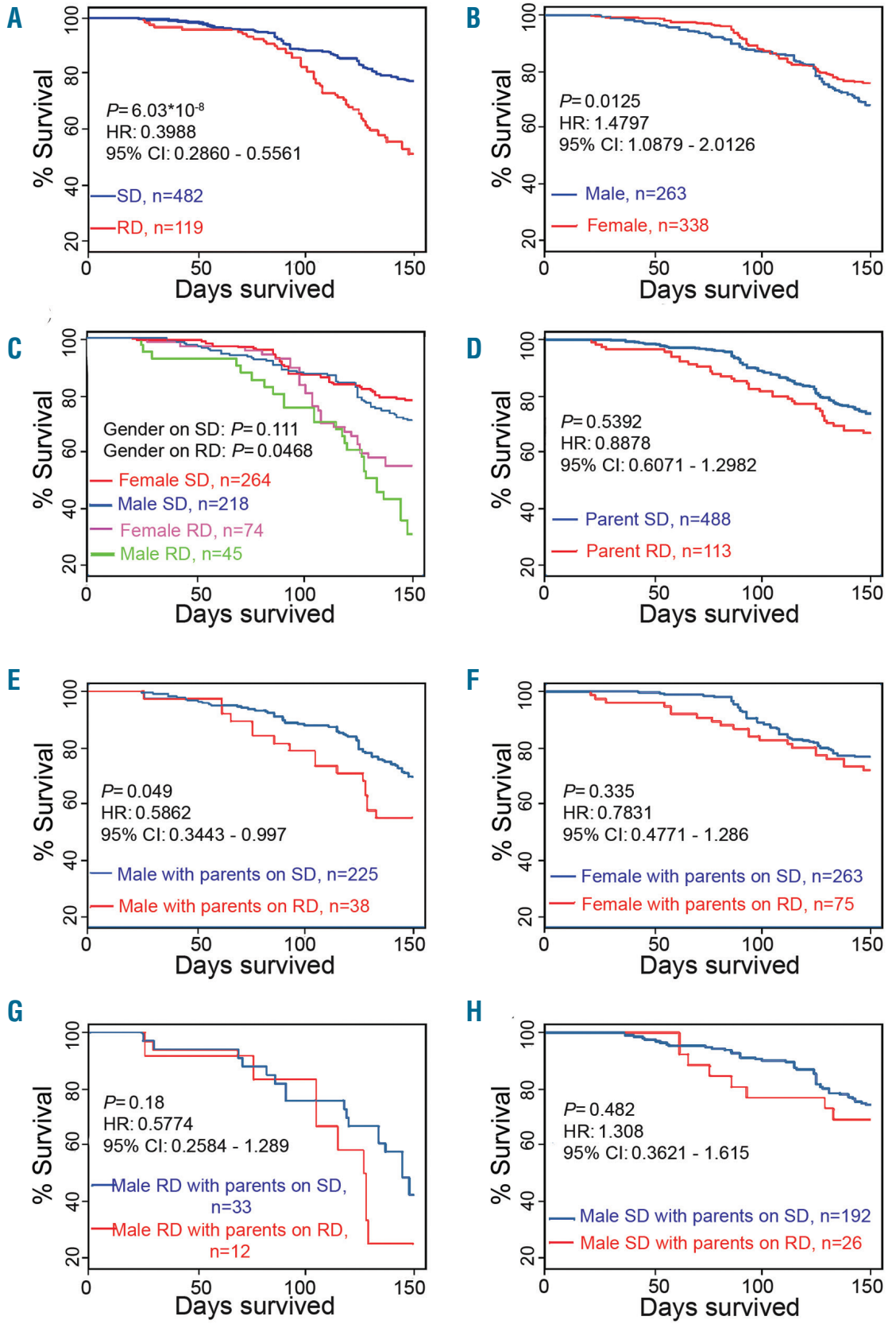

Figure 2. Diet and gender affect the survival of sickle mice. Transgenic homozygous Berkley mice expressing $>99 \%$ human sickle hemoglobin were used. The protein, fat, mineral, vitamin, amino acid, and omega-3-fatty acid contents were higher in the sickle diet (SD) than in the regular diet (RD). Pups were weaned 3 weeks after birth, fed the SD for 10 days after weaning and then randomly assigned to either the SD or RD until 150 days of age. Multivariate analysis was performed on the relationship between rates of survival and pup gender, pup diet, and parental diet. (A) Survival of pups fed the SD or RD. (B) Survival of male and female pups. (C) Survival of male and female pups fed the RD or SD. (D) Survival of pups born to parents fed the SD or RD. (E) Survival of male pups on the SD or RD. (F) Survival of female pups on the SD or RD. (G) Survival of male pups fed the RD and born to parents fed either the SD or RD. $(H)$ Survival of male pups fed the SD born from parents fed either the SD or RD. HR: hazard ratio; $95 \% \mathrm{Cl}$ : 95\% confidence interval. 
diets. At 150 days, $76 \%$ of females and $68 \%$ of males were alive (Figure 2B). In multivariate analysis, females survived significantly longer than males (HR: 1.4797; 95\% CI: 1.0879-2.0126; $P=0.0125)$. We then studied the influence of diet on gender-specific survival of $\mathrm{HbSS}$ BERK mice (Figure 2C). Compared to the RD, the SD significantly increased the survival of both males (HR: $0.3411 ; 95 \%$ CI: $\left.0.2167-0.5367 ; P=3.3 \times 10^{-6}\right)$ and females (HR: $0.4424 ; 95 \%$ CI: $0.2821-0.6939 ; P=3.8 \times 10^{-4}$ ). There was not a statistically significant difference between the survival of females and males on the SD (HR: 1.357; 95\% CI: 0.9318-1.976; $P=0.111$ ), while there was a significant difference between the survival of males and females on the RD (HR: 1.687; 95\% CI: 1.008-2.825; $P=0.0468$ ).

Several gender-specific differences may explain the longer survival of females than males. Blood from female donors has lower levels of hemolysis than blood from male donors in the SCD population, ${ }^{16}$ which may be due to the effects of gender on hemoglobin $\mathrm{F}$ levels, ${ }^{17}$ red blood cell membrane stability, ${ }^{18}$ and nitric oxide bioavailability. ${ }^{19}$ Our observations in sickle mice are complementary to those in a long-term follow up of about 3,800 SCD patients with a median survival of 42 and 48 years in males and females, respectively. ${ }^{20}$ The Walk-PHaSST study showed a progressively higher proportion of female SCD patients with increasing age, with a lower degree of hemolysis and vasculopathy, a reason for their prolonged survival. ${ }^{21}$ Our observations of improved survival of male mice given an enriched diet suggest the significance of dietary interventions in improving survival of males with SCD.

To determine the effect of parents' diet during gestation on their offsprings' survival, we randomly assigned 601 pups born to SD-fed and RD-fed parents to the SD (488 pups) or the RD (113 pups) (Figure 1). At 150 days, $73 \%$ of pups of SD-fed parents and $68 \%$ of pups of RDfed parents were alive (Figure 2D). Pups born to parents fed the SD showed a trend towards increased survival, although parental diet did not have a statistically significant impact on survival of pups of both genders together (HR: 0.8878; 95\% CI: 0.6071-1.2982; P=0.5392) (Figure 2D). However, male pups with SD-fed parents survived significantly longer than male pups with $\mathrm{RD}$-fed parents (HR: 0.5862; 95\% CI: 0.3443-0.997; $P=0.049$ ) (Figure 2E). The survival of female pups with parents fed the SD was similar to that of female pups with parents fed the $\mathrm{RD}$ (HR: 0.7831; 95\% CI: 0.4771-1.286; $P=0.335$ ) (Figure 2F). Controlling for diet, we found that parental diet did not statistically significantly affect the survival of male pups on the $\mathrm{SD}$ or $\mathrm{RD}$, but a trend towards improved survival of male pups on the $\mathrm{SD}$ born to parents fed the $\mathrm{RD}$ was observed (Figure 2G,H).

Maternal diet during gestation and lactation influences long-term physical and mental development of the offspring. Suboptimal maternal diet alters mu opioid receptor and dopamine type 1 receptor binding and affects the inflammatory gene expression profile and behavioral responses to stressors in offspring. ${ }^{22}$ Intrauterine inflammation led to gender-specific effects on neuroinflammation and behavior in rodents. ${ }^{23}$ Since inflammation plays a critical role in the pathobiology of SCD, it is likely that the effects of the dietary modulations in our study may be mediated via modulation of inflammation and its consequences. Our data provide a strong rationale for analyzing the mechanisms underlying improved survival with dietary interventions.

SCD demonstrates the features of disease-related malnutrition with inflammation according to the guidelines for malnutrition from the European Society of Clinical
Nutrition and Metabolism (ESPEN). ${ }^{24}$ Disease-related malnutrition coupled with social malnutrition requires attention in resource-limited settings, which may have an additive effect on observed mortality in SCD. Our observations provide the proof of principle that targeting malnutrition with dietary interventions can improve survival. Meta-analyses of multiple prospective studies have shown that food-based dietary manipulations to provide combined increases of nitric oxide, anti-oxidants and vitamins, etc. had significant beneficial effects on reducing the risk of cardiovascular disease and all-cause mortality compared to those associated with the intake of individual supplements. ${ }^{25,26}$ Therefore, considering the beneficial effect of several individual supplements, including arginine and glutamine on SCD pathobiology, and our observations that dietary interventions improved the survival of sickle mice, further examination of the mechanisms and effectiveness of individual supplements, compared to an enriched diet, on survival in SCD is required. The survival of male pups was prolonged by giving their parents the $\mathrm{SD}$; this novel observation is potentially clinically significant and warrants further investigation into gender-specific effects of dietary interventions.

Om B. Jahagirdar, ${ }^{1 *}$ Aditya M. Mittal, ${ }^{1 *}$ Waogwende L. Song-Naba, Ritu Jha, ${ }^{1}$ Stacy B. Kiven, Susan T. Thompson, 'John E Connett ${ }^{2}$ and Kalpna Gupta ${ }^{1}$

${ }^{*} O B J$ and $A M M$ contributed equally to this manuscript.

"Vascular Biology Center, Division of Hematology, Oncology \& Transplantation, Department of Medicine, University of Minnesota, Minneapolis, MN and ${ }^{2}$ School of Public Health, Division of Biostatistics, University of Minnesota, Minneapolis, MN, USA.

Funding: UO1HL117664. National Heart, Lung, and Blood Institute.

Acknowledgments: the authors thank Barb Benson and

Michael J. Franklin for proof reading the manuscript.

Correspondence: KALPNA GUPTA.

gupta014@umn.edu

doi:10.3324/haematol.2018.208322

Information on authorship, contributions, and financial \& other disclosures was provided by the authors and is available with the online version of this article at www. haematologica.org.

\section{References}

1. Makani J, Cox SE, Soka D, et al. Mortality in sickle cell anemia in Africa: a prospective cohort study in Tanzania. PLoS One. 2011;6(2):e14699.

2. Tewari S, Rees D. Morbidity pattern of sickle cell disease in India: a single centre perspective. Indian J Med Res. 2013;138(3):288-290.

3. Dekker LH, Fijnvandraat K, Brabin BJ, van Hensbroek MB. Micronutrients and sickle cell disease, effects on growth, infection and vaso-occlusive crisis: a systematic review. Pediatr Blood Cancer. 2012; 59(2):211-215.

4. Barden EM, Zemel BS, Kawchak DA, Goran MI, Ohene-Frempong $\mathrm{K}$, Stallings VA. Total and resting energy expenditure in children with sickle cell disease. J Pediatr. 2000;136(1):73-79.

5. Paszty C, Brion CM, Manci E, et al. Transgenic knockout mice with exclusively human sickle hemoglobin and sickle cell disease. Science. 1997;278(5339):876-878.

6. Sagi V, Song-Naba WL, Benson BA, Joshi SS, Gupta K. Mouse models of pain in sickle cell disease. Curr Protoc Neurosci. 2018;85(1):e54.

7. Capers PL, Hyacinth HI, Cue S, et al. Body composition and grip strength are improved in transgenic sickle mice fed a high-protein diet. J Nutr Sci. 2015;4:e6.

8. Manci EA, Hyacinth HI, Capers PL, et al. High protein diet attenuates histopathologic organ damage and vascular leakage in transgenic murine model of sickle cell anemia. Exp Biol Med (Maywood). 2014; 239(8):966-974.

9. Daak AA, Elderdery AY, Elbashir LM, et al. Omega 3 (n-3) fatty acids 
LETTERS TO THE EDITOR

down-regulate nuclear factor-kappa B (NF-kappaB) gene and blood cell adhesion molecule expression in patients with homozygous sickle cell disease. Blood Cells Mol Dis. 2015;55(1):48-55.

10. Dak AA, Ghebremeskel K, Masan Z, et al. Effect of omega-3 (n-3) fatty acid supplementation in patients with sickle cell anemia: randomized, double-blind, placebo-controlled trial. Am J Chin Nutr. 2013;97(1):37-44

11. Zemel BS, Kawchak DA, Fund EB, Ohene-Frempong K, Stallings VA. Effect of zinc supplementation on growth and body composition in children with sickle cell disease. Am J Chin Nutr. 2002;75(2):300-307.

12. Lindenbaum J, Klipstein FA. Folic acid deficiency in sickle-cell anemia. N Encl J Med. 1963;269:875-882.

13. Raul DK, Zhang X, Dasgupta T, Fabre ME. Arginine therapy of transgenic-knockout sickle mice improves microvascular function by reducing non-nitric oxide vasodilators, hemolysis, and oxidative stress. Am J Physiol Heart Circ Physiol. 2008;295(1):H39-47.

14. Morris CR, Kuypers FA, Lavrisha L, et al. A randomized, placebocontrolled trial of arginine therapy for the treatment of children with Haematological. 2013;98(9):1375-1382.

15. Niihara Y, Miller ST, Ranter J, et al. A phase 3 trial of L-glutamine in sickle cell disease. N Angl J Med. 2018;379(3):226-235.

16. Manias T, Sinchar D, Osei-Hwedieh D, et al. Testosterone-dependent sex differences in red blood cell hemolysis in storage, stress, and disease. Transfusion. 2016;56(10):2571-2583.

17. Dover GJ, Smith KD, Chang YC, et al. Fetal hemoglobin levels in sickle cell disease and normal individuals are partially controlled by an X-linked gene located at Xp22.2. Blood. 1992;80(3):816-824. 18. Jordan A, Chen D, Mi OL, Manias T, Gladwin MT, Asker JP. Assessing
the influence of component processing and donor characteristics on quality of red cell concentrates using quality control data. Vax Sang. 2016;111(1):8-15

19. Gladwin MT, Schechter AN, Ognibene FP, et al. Divergent nitric oxide bioavailability in men and women with sickle cell disease. Circulation. 2003;107(2):271-278.

20. Plat OS, Brambilla DJ, Rose WF, et al. Mortality in sickle cell disease. Life expectancy and risk factors for early death. N Angl J Med. 1994; 330(23):1639-1644.

21. Saraf SL, Manias T, Zhang X, et al. Reduced hemolysis and related complications in females with sickle cell disease. Blood. 2017;130( Suppl 1):3530.

22. Thanes PK, Zhuo J, Robison L, et al. Suboptimal maternal diets alter $\mathrm{mu}$ opioid receptor and dopamine type 1 receptor binding but exert no effect on dopamine transporters in the offspring brain. Int J Lev Neurosci. 2018;64:21-28. 23. Makinson R, Lloyd K, Rayasam A, et al. Intrauterine inflammation
induces sex-specific effects on neuroinflammation, white matter, and behavior. Brain Behav Immun. 2017;66:277-288.

24. Cederholm T, Barazzoni R, Austin P, et al. ESPEN guidelines on definitions and terminology of clinical nutrition. Chin Nutr. 2017;36(1):49-64

25. Ane D, Geum N, Giovannucci E, et al. Dietary intake and blood concentrations of antioxidants and the risk of cardiovascular disease, total cancer, and all-cause mortality: a systematic review and dose2018;108(5):1069-1091.

26. Aggarwal M, Bozkurt B, Panjrath $G$, et al. Lifestyle modifications for preventing and treating heart failure. J Am Coll Cardiol. 2018; 72(19):2391-2405.

haematologica 2019; 104:e334 